\title{
miR-145 overexpression suppresses the migration and invasion of metastatic melanoma cells
}

\author{
PETER DYNOODT $^{1}$, REINHART SPEECKAERT ${ }^{1}$, OLIVIER DE WEVER ${ }^{2}$, INÈS CHEVOLET ${ }^{1}$, \\ LIEVE BROCHEZ $^{1}$, JO LAMBERT ${ }^{1}$ and MIREILLE VAN GELE ${ }^{1}$ \\ ${ }^{1}$ Department of Dermatology and ${ }^{2}$ Laboratory of Experimental Cancer Research, Department of Radiotherapy \\ and Nuclear Medicine, Ghent University Hospital, 9000 Ghent, Belgium
}

Received October 17, 2012; Accepted December 7, 2012

DOI: $10.3892 /$ ijo.2013.1823

\begin{abstract}
MicroRNAs (miRNAs) are post-transcriptional modulators of gene expression which play important roles in tumorigenesis and cancer metastasis. Since they are often highly deregulated in various types of cancer, miRNAs may be effective treatment targets. miRNA profiling studies of melanoma have led to the identification of several tumor suppressor miRNAs. One of these include miR-145, although functional data proving its specific function are limited. Therefore, in this study, we examined the expression levels of miR-145 in three melanoma cell lines (BLM, FM3P and WM793). Additional gain-of-function experiments revealed that miR-145 exerts an anti-proliferative effect in the primary, non-invasive melanoma cell line, WM793, whereas cell migration and the invasive potential of metastatic melanoma cells was suppressed following transfection with miR-145 mimics. In order to investigate the mechanisms by which miR-145 exerts its invasion suppressor function, we examined the expression level of target genes [fascin homolog 1 (FSCN1), myosin-Va (MYO5A and $S O X 9$ ] and that of an indirect target (RAB27A) following the overexpression of miR-145. The results showed that $S O X 9$, $M Y O 5 A$ and $R A B 27 A$ were not involved in the biological effects caused by miR-145 mimics. Surprisingly, we discovered that miR-145 in melanoma, in contrast to many other tumor types, does not necessarily act via the target, FSCN1, since the downregulation of FSCN1 did not inhibit cell proliferation or migration but, on the contrary, increased cell invasion in two out of the three melanoma cell lines examined. Our in vitro data is in accordance with previously reported in vivo data describing the low expression of FSCN1 in malignant melanomas when compared to dysplastic nevi, suggesting that the expression of FSCN1 decreases as the formation and progression stage of
\end{abstract}

Correspondence to: Dr Mireille Van Gele, Department of Dermatology, Ghent University Hospital, De Pintelaan 185, 9000 Ghent, Belgium

E-mail: mireille.vangele@ugent.be

Key words: miR-145, suppressor of invasion, melanoma, fascin homolog 1 melanoma advances. In conclusion, our data provide evidence that miR-145 is an invasion suppressor in metastatic melanoma cells. Despite the fact that it remains unclear which genes or pathways are regulated by miR-145 in melanoma, miR-145 may serve as a useful therapeutic agent in melanoma when re-expressed in situ.

\section{Introduction}

The incidence of melanoma worldwide has been increasing more rapidly than any other type of cancer over the past few decades (1). It mainly affects young populations and has become the fifth most common type of cancer in females. Compared to other skin cancers, such as basal cell carcinoma and squamous cell carcinoma, melanoma has a higher metastatic potential. Therefore, early detection is essential, since complete surgical removal is the only treatment with a high probability of complete recovery for early-stage melanoma. Patients with regional and distant metastatic disease have a much worse prognosis and current treatments, even novel therapeutic options, have limited effects on overall survival. This emphasizes the need to continue the research for more effective treatment modalities.

The development and progression of malignant melanoma has been associated with genetic changes, deregulated signaling pathways and changes in the tumor microenvironment $(2,3)$. Apart from the identification of affected genes, transcription factors and epigenetic changes in regulatory genes, the detailed molecular analysis of melanoma cell lines and tumor samples at different stages has also assigned a role for microRNAs (miRNAs) in the development, progression and invasiveness of melanoma. Recently, several profiling experiments have identified numerous differentially regulated miRNAs that are associated with the early and late progression of malignant melanoma, providing a series of candidate miRNAs to be analyzed for their potential as diagnostic markers or therapeutic targets (4-6). One of these, miR-145, seems to be associated with the early progression of melanoma, since its expression is upregulated in primary melanoma cell lines in contrast to normal human epidermal melanocytes (NHEMs) (5). A previous study demonstrated that miR-145 was upregulated in two primary melanoma tissue samples, as compared to NHEMs, and two melanoma cell lines with 
different metastatic potential (7). By profiling a large panel of melanoma tissue samples, Segura et al (8) defined a melanoma miRNA signature capable of predicting post-recurrence survival in metastatic melanoma. Of note, this study revealed that a higher expression of miR-145 was associated with longer patient survival. This finding is consistent with previous reports on lung, colon, breast and prostate cancer, suggesting a tumor suppressor role for miR-145 $(9,10)$.

The exact function or mechanism of miR-145 in malignant melanoma is unclear. To date, only one study has investigated the effect of the ectopic expression of miR-145 in melanoma, using two canine and two human melanoma cell lines (11). Depending on the cell lines under study, the overexpression of miR-145 inhibited cell growth and/or cell migration, suggesting that miR-145 acts as a tumor suppressor in both canine and human malignant melanomas. In the present study, we aimed to collect additional evidence to sustain the hypothesis that miR-145 is a melanoma tumor suppressor by investigating its possible involvement, not only in cell proliferation and migration, but also in cell invasiveness. miR-145 was overexpressed in three human melanoma cell lines, and its effect on cell growth, migration and invasion, as well as on the expression of a number of target genes was evaluated.

\section{Materials and methods}

Cell culture. In this study, we chose three different melanoma cell lines with a decreasing metastatic potential (BLM, FM3P and WM793). The highly metastatic melanoma cell line, BLM, was originally obtained from Dr Leon Van Kempen (Department of Biochemistry, Nijmegen, The Netherlands) and cultured in Dulbecco's modified Eagle's medium (DMEM) (Life Technologies Europe B.V., Ghent, Belgium) supplemented with $10 \%$ fetal calf serum (FCS), $200 \mu \mathrm{M}$ L-glutamine, $50 \mu \mathrm{g} / \mathrm{ml}$ streptomycin, $50 \mathrm{U} / \mathrm{ml}$ penicillin and Fungizone. The metastatic melanoma cell line, FM3P (12), was cultured in RPMI-1640 medium (Life Technologies Europe B.V.) supplemented with $10 \%$ FCS, $200 \mu \mathrm{M}$ L-glutamine, $50 \mu \mathrm{g} / \mathrm{ml}$ streptomycin, $50 \mathrm{U} / \mathrm{ml}$ penicillin and Fungizone. The melanoma cell line, WM793, was kindly provided by Dr Carola Berkling (Department of Dermatology, Ludwig Maximilian University of Munich, Munich, Germany). This cell line was derived from a primary melanoma (stage I) in the sternal area and cultured in MCDB153:Leibovitz L-15 (4:1) medium supplemented with $2 \% \mathrm{FCS}, 1.68 \mathrm{mM} \mathrm{CaCl}_{2}, 5 \mu \mathrm{g} / \mathrm{ml}$ insulin, $50 \mu \mathrm{g} / \mathrm{ml}$ streptomycin, $50 \mathrm{U} / \mathrm{ml}$ penicillin and Fungizone (information is also available at http://ccr.coriell.org, catalog ID WC00062). Human primary epidermal melanocyte cultures were established as previously described $(13,14)$. All cells were incubated at a temperature of $37^{\circ} \mathrm{C}, 99 \%$ humidity and $10 \% \mathrm{CO}_{2}$.

RNA isolation and real-time quantitative PCR. Total RNA, including miRNAs, was extracted from the melanoma cell lines and melanocytes (pooled from three different donors) using the miRNeasy Mini kit (Qiagen, Venlo, The Netherlands) according to the manufacturer's recommendations. A DNase treatment was performed and first-strand cDNA was generated by reverse transcription using the iScript cDNA synthesis kit according to the manufacturer's instructions (Bio-Rad, Eke, Belgium). Relative gene expression levels were determined using a SYBR-Green I reverse transcription-PCR assay as described by Vandesompele et al (15) and the comparative $\mathrm{Cq}$ method was used for quantification. PCR reactions were performed by using SYBR ${ }^{\circledR}$-Green I master mix (Eurogentec, Ougrée Seraing, Belgium) and were run on a MyiQ ${ }^{\mathrm{TM}}$ iCycler (Bio-Rad). To correct for differences in RNA quantities and cDNA synthesis efficiency, relative gene expression levels were normalized using the geometric mean of three reference genes ( $R P L 13 A, U B C$ and $S D H A$ ), as described previously by Vandesompele et al (16).

Pre-miR/short interfering RNA (siRNA) transfections and miRNA quantification. To achieve the upregulation of miR-145, chemically modified double-stranded (ds) nucleic acids were transfected with minimal cellular stress to mimic endogenous mature miRNAs (pre-miR-145, PM11480; Life Technologies Europe B.V.). This enabled the detailed analysis of the biological effects of miRNAs via gain-of-function experiments. Pre-miR negative control 2 (AM17111; Life Technologies Europe B.V.) is a random-sequence pre-miR molecule that has been extensively tested in cell lines and tissues and validated to not produce identifiable effects on known miRNA function. To induce the knockdown of fascin homolog 1 (FSCN1), validated synthetic siRNA molecules were used, obtained from Qiagen, Hilden, Germany (Hs_FSCN1_3 Flexitube siRNA, SI00421806). A scrambled sequence (5'-AUUAUCUAGGAGAUAUCAC-3'), showing no homology to any known gene, was used as the negative control (Eurogentec). For qPCR and western blot analysis, melanoma cells were plated into $60-\mathrm{mm}$ dishes (or 6-well plates) at a density of 500,000 per dish/well in their specific medium, depending on the cell line used. Twenty-four hours later the medium was replaced with $2.4 \mathrm{ml}$ of fresh medium. The final pre-miR-145 or siRNA concentration was $50 \mathrm{nM}$ following the addition of $18 \mu \mathrm{l}$ HiPerFect Transfection Reagent (Qiagen) to $100 \mu \mathrm{l}$ of serum-free culture medium. This solution was mixed by vortexing and incubated for $10 \mathrm{~min}$ at room temperature to allow the formation of transfection complexes. These complexes were added in a dropwise manner onto the cells. The medium was replaced $24 \mathrm{~h}$ later and gene silencing was monitored at the desired time points by harvesting the cells for either RNA isolation or for preparation of whole-cell lysates (see below). All transfections were performed in triplicate.

Quantification of miR-145 by TaqMan Real-Time PCR was carried out as described by the manufacturer (Life Technologies Europe B.V.). Briefly, $800 \mathrm{ng}$ of template RNA was reverse-transcribed using the TaqMan MicroRNA Reverse Transcription kit and the multiplex RT primer pools containing miRNA-specific stem-loop primers. An RT-product diluted five times was introduced into a 5- $\mu \mathrm{l}$ PCR reaction. Reactions were run in 384-well plates on a 7900HT RT-qPCR system (Applied Biosystems Europe, Halle, Belgium) at $95^{\circ} \mathrm{C}$ for $10 \mathrm{~min}$, followed by 40 cycles at $95^{\circ} \mathrm{C}$ for $15 \mathrm{sec}$ and $60^{\circ} \mathrm{C}$ for 1 min. miR-145 expression was normalized between different samples based on the geometric means of the expression values of three small nucleolar RNAs (U43, U48 and U49) (Life Technologies Europe B.V.).

Western blot analysis. Lysates of melanoma cells or pooled melanocytes $(n=3)$ were produced and immunoblotting was 
performed as previously described by Van Gele et al (14). Primary antibodies included the purified rabbit anti-myosin-Va (MYO5A) exon F $(1 / 10,000)(14)$, a rabbit polyclonal antibody against RAB27A (H-60) (1/200, sc-22756, Santa Cruz, Heidelberg, Germany), a polyclonal antibody against FSCN1 (IM20) (1/200, ab49815, Abcam, Cambridge, UK), a rabbit polyclonal antibody against GAPDH (G9545) (1/10,000, Sigma-Aldrich, Bornem, Belgium) and a mouse monoclonal antibody anti- $\alpha$-tubulin clone B-5-1-2 (T5168) (1/8,000, Sigma-Aldrich). The latter was used to control loading. The blots were incubated with the appropriate HRP-conjugated secondary antibody (1/3,000, Amersham Biosciences Ltd., Buckinghamshire, UK). Detection was performed with an ECL detection system kit (Amersham Biosciences, Ltd.).

Cell growth, wound healing and invasion assays. All three melanoma cell lines were transfected with pre-miR-145 or siFSCN1 and their respective negative controls, as described above, in order to examine the effect on cell proliferation, cell migration and invasion. All experiments were performed in triplicate. Cell growth was determined using the trypan blue exclusion test of cell viability. Viable cells were counted $48 \mathrm{~h}$ after transfection, while non-viable cells containing a blue cytoplasm were excluded. Cell migration activity was evaluated by wound healing migration assays. Cells were plated in six-well tissue culture dishes and grown to confluence. The confluent cell monolayer was scraped with a plastic pipette tip of $1 \mathrm{~mm}$ diameter which was followed by the addition of fresh medium, specific for each cell line. On the exterior bottom side of each dish, a mark was made at six arbitrary places where the width of the wound was measured with an inverted microscope (objective $\mathrm{x} 4$ ) at the time of wound induction (initiation, $0 \mathrm{~h}$ ) and after $12 \mathrm{~h}$ of incubation at $37^{\circ} \mathrm{C}$. Migration was expressed as the mean \pm SEM of the difference between the measurement at wound initiation and at $12 \mathrm{~h}$. The results of the controltransfected cells were rescaled to $100 \%$.

Cell invasion assays were performed by the use of collagen type I matrices, as described by De Wever et al (17). Briefly, six-well tissue culture dishes were filled with $1.35 \mathrm{ml}$ of neutralized type I collagen (BD Biosciences, Erembodegem, Belgium) and incubated overnight at $37^{\circ} \mathrm{C}$ to allow gelling. Cells were harvested using PBS buffer and trypsin/EDTA and seeded on top of the collagen gels. Cultures transfected with specific siRNAs or miRNAs were incubated for $24 \mathrm{~h}$ at $37^{\circ} \mathrm{C}$. The cell invasion index (cells with invasive extensions versus the total number of cells) was calculated by manually counting the number of invading and non-invading cells present in ten independent microscopic fields. The invasion index of the control-transfected cells was rescaled to $100 \%$. Statistical significance was determined by the Mann-Whitney test by use of SPSS version 19.0 software (IBM).

\section{Results}

miR-145 expression in melanoma cell lines and human primary melanocytes. We first determined the expression level of miR-145 in three melanoma cell lines and in pooled NHEMs from three donors. The expression of miR-145 was extremely low in the two metastatic melanoma cell lines (BLM and FM3P) and slightly, but not significantly, increased

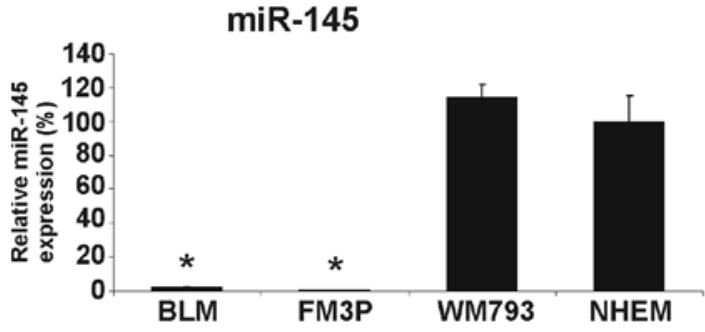

Figure 1. miR-145 expression in melanoma cell lines and melanocytes. The relative expression level of miR-145 was determined by real-time qPCR in BLM, FM3P, WM793 cells and normal human primary melanocytes (NHEMs) derived from three different donors. The mean \pm SEM of three independent experiments is shown. P-values were determined with an unpaired t-test $\left({ }^{*} \mathrm{P}<0.001\right)$.

in the primary melanoma cell line, WM793, compared to the NHEMs (Fig. 1). The downregulation of miR-145 in the metastatic cancer cell lines was in accordance with the role of miR-145 as a tumor suppressor miRNA in different types of cancer, including melanoma $(8,11)$.

Effect of miR-145 expression on cell growth, invasion and migration activity in melanoma cell lines. To investigate the functional role of miR-145 in the melanoma cell lines, we performed gain-of-function experiments by transfecting miR-145 mimics into each melanoma cell line. The overexpression of miR-145 resulted in decreased cell growth compared to the negative control-transfected cells (NC2) only in the WM793 cell line (Fig. 2A). Wound healing assays showed significant cell migration inhibition in the miR-145 transfectants compared to the controls in the BLM and WM793 cells (\% inhibition: BLM, 24 $\pm 1.4 \%$ SEM and WM793, 47 $\pm 1.6 \%$ SEM $)(\mathrm{P}<0.001)$, whereas in the FM3P cells, only a slight suppression of migration was observed (Fig. 2B).

The collagen I invasion assay also showed a significant inhibition of cell invasion when miR-145 was overexpressed in the BLM $(68 \pm 4 \%$ SEM) $(\mathrm{P}<0.001)$ and FM3P $(55 \pm 1 \%$ SEM $)$ $(\mathrm{P}<0.01)$ cell lines (Fig. 3). No inhibitory effect was observed in the miR-145 transfectants in the primary WM793 cell line.

Effect of miR-145 overexpression on (potential) target genes in melanoma cell lines. We overexpressed miR-145 in all three cell lines and measured the effect of miR-145 on a number of (potential) target genes (FSCN1, MYO5A and SOX9) and on an indirect target $(R A B 27 A)(18)$. Transfection experiments were performed in triplicate with miR-145 mimic ds oligonucleotides (pre-miR-145) and a negative control miRNA. A significant decrease was observed for the target genes, $M Y O 5 \mathrm{~A}$ and $F S C N 1$, in the BLM cells $(52 \pm 5 \%$ SEM and $64 \pm 5 \%$ SEM, respectively) $(\mathrm{P}<0.001)$ and in the WM793 cells $(73 \pm 2 \%$ SEM and $52 \pm 3 \%$ SEM, respectively) $(\mathrm{P}<0.001)$ (Fig. 4A and B). The expression levels of MYO5A and FSCN1 were also decreased in the FM3P cells, although not to a significant extent (Fig. 4C). The expression of $R A B 27 A$ was significantly reduced in the BLM cells $(\mathrm{P}<0.05)$ but not in the WM793 and FM3P cells. There were no significant differences observed in SOX9 expression among the three examined cell lines.

Since FSCN1 is a well-known target gene of miR-145 in many different types of cancer (19-21), we examined via western 


\section{A Cell proliferation assay}
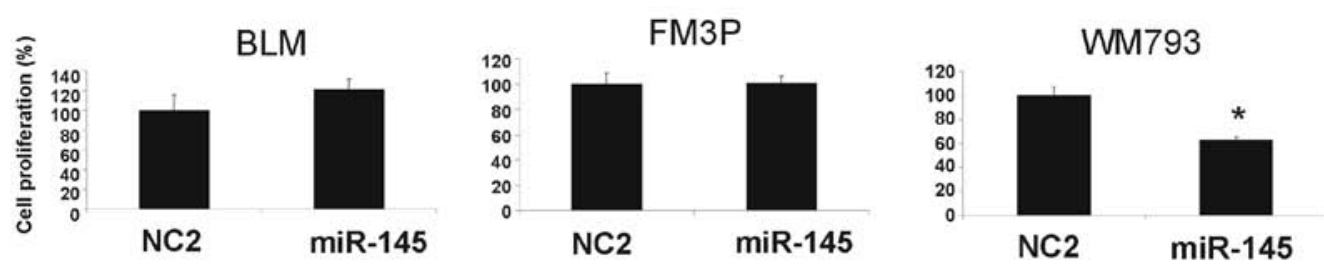

B

Wound healing assay

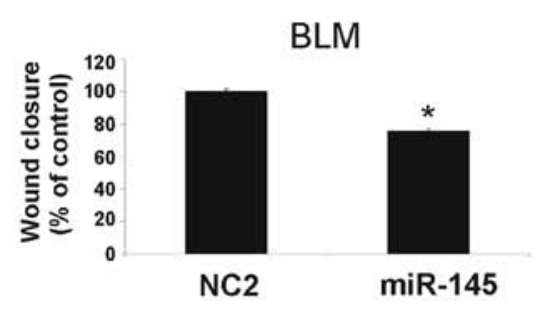

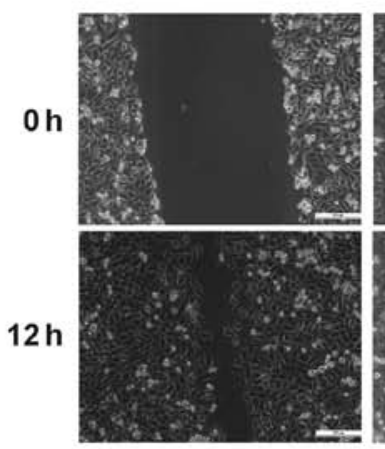

NC2
BLM

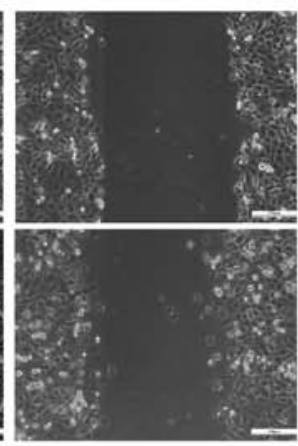

$\operatorname{miR}-145$

FM3P

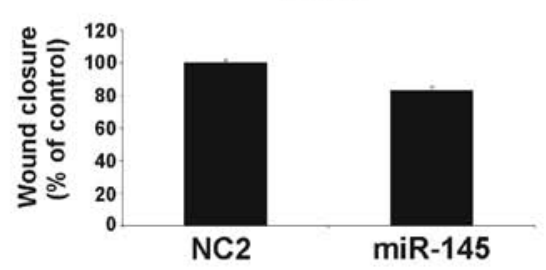

WM793
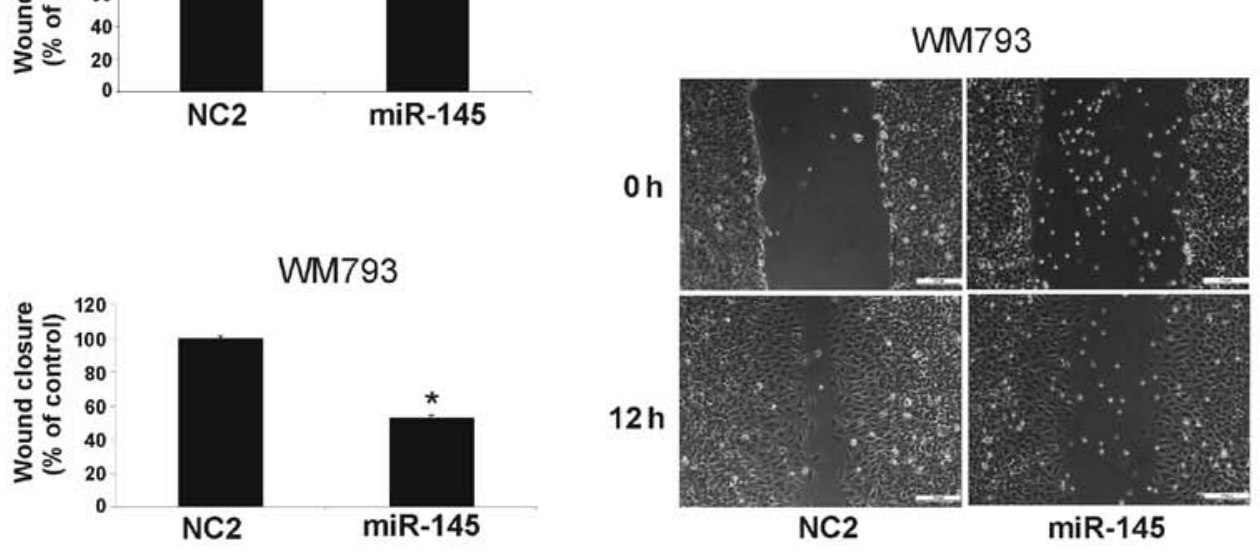

Figure 2. Effect of miR-145 overexpression on cell growth and cell migration. (A) Cell proliferation was only reduced in WM793 cells following transfection with miR-145 versus negative control (NC2) (60 h post-transfection). (B) Cell migration activity was determined by the wound healing assay. The results of the migration assay are presented as histograms (left) and as phase contrast images for BLM and WM793 cells (right panel) (scale bar, 200 $\mu \mathrm{m}$ ). In all histograms, mean levels of three independent experiments \pm SEM are depicted. P-values were determined with an unpaired t-test ( $\mathrm{P}<0.001)$.

blot analysis whether the ectopic expression of miR-145 reduced FSCN1 protein levels. A reduction in FSCN1 expression was observed following the overexpression of miR-145, compared to the negative control conditions in all three examined cell lines (Fig. 4D).

Effect of FSCN1 knockdown on cell growth, invasion and migration activity in melanoma cell lines. To further examine the mechanism by which miR-145 exerts its invasion suppressor role in our studied melanoma cell lines, we decided to knock down FSCN1 and study this effect by functional assays. After the transfection of siFSCN1 into the melanoma cell lines we determined its expression at the mRNA and protein levels.
Real-time qPCR revealed a significant reduction in FSCN1 expression at the mRNA level $[62 \pm 0.3 \%$ SEM $(\mathrm{P}<0.001)$, $39 \pm 6 \%$ SEM $(\mathrm{P}<0.01)$ and $58 \pm 1 \%$ SEM $(\mathrm{P}<0.001)$, in $\mathrm{BLM}$, FM3P and WM793 cells, respectively] (Fig. 5A). The silencing of FSCN1 was also confirmed at the protein level by western blot analysis (Fig. 5B). The knockdown of FSCN1 did not result in a significantly reduced cell growth (Fig. 6A) or the inhibition of migration in any of the transfected cell lines. Fig. 6B depicts the cell migration results for the BLM cell line. Surprisingly, the silencing of FSCN1 resulted in a significant increase in cell invasion in two out of the three studied melanoma cell lines (BLM, 45 $\pm 1 \%$ SEM increase; and WM793, 40 $\pm 3 \%$ SEM increase) $(\mathrm{P}<0.001)$ (Fig. 7). These results led to the conclusion 
Invasion assay
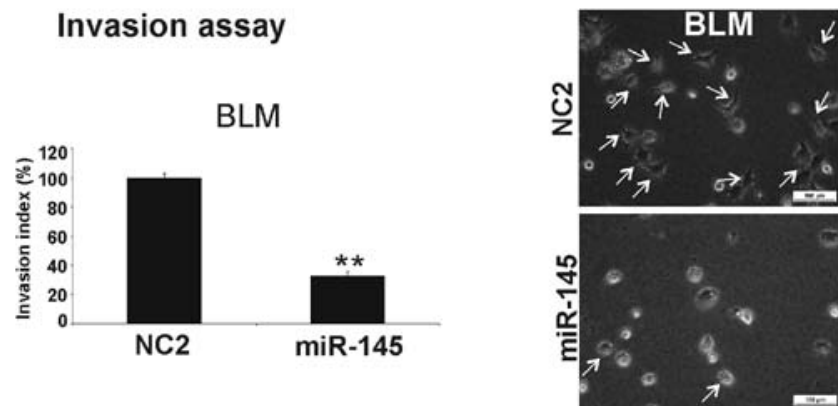

FM3P

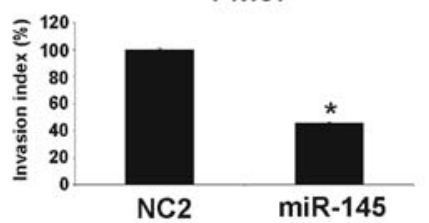

WM793

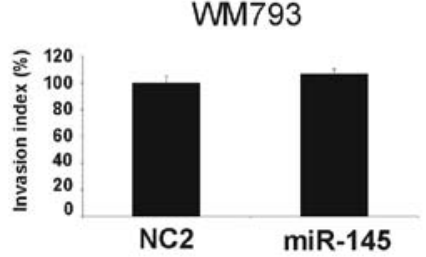

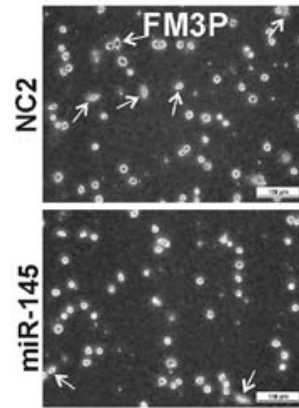

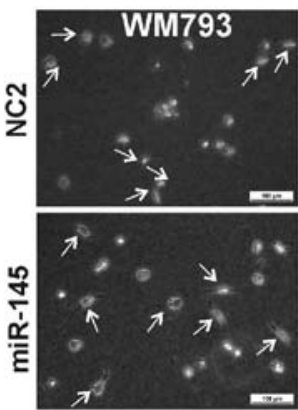

Figure 3. miR-145 suppresses the invasion of metastatic melanoma cell lines. Invasion assays were performed following the transfection of miR-145 and the negative control (NC2) into melanoma cell lines. The invasion index (\%), which is the ratio of the number of cells containing invasive extensions over the total number of cells counted in each field (for a total a 10 fields) is depicted on the Y-axis of each histogram (left). The invasion index for each NC2 condition has been rescaled to $100 \%$. Phase contrast images are shown on the right panel. Arrows indicate invasive extensions (scale bar, $100 \mu \mathrm{m}$ ). P-values were determined by the Mann-Whitney test $\left({ }^{* *} \mathrm{P}<0.001\right.$ and $\left.^{*} \mathrm{P}<0.01\right)$.

that the invasion suppressor function of miR-145 is possibly caused by other target gene(s) or pathways.

MYO5A may be another possible target gene, since its mRNA expression was also reduced after the overexpression of miR-145 in our melanoma cell lines (Fig. 4A-C). However, when examining the protein expression levels of MYO5A and RAB27A by western blot analysis in the BLM, FM3P and WM793 cells, we detected only a moderate protein expression of MYO5A and a very low expression level of RAB27A in the FM3P cells as compared to NHEMs, while neither protein was expressed in the other two cell lines, even following long exposure times (data not shown). Due to the low or absent expression of MYO5A in our studied melanoma cell lines we could not further examine the effect of MYO5A (or RAB27A) knockdown by functional assays. Based on these results, however, we assumed that miR-145 does not exert its invasion suppressor activity through the direct targeting of MYO5A in our melanoma cell lines.

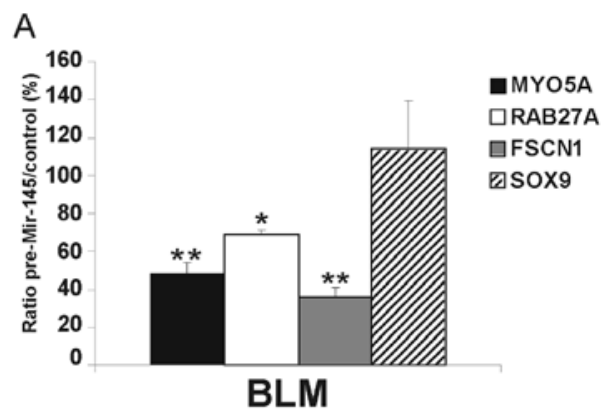

B

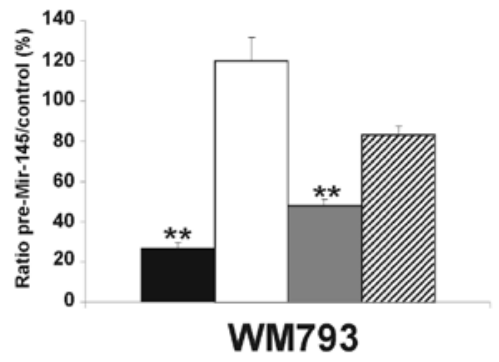

C
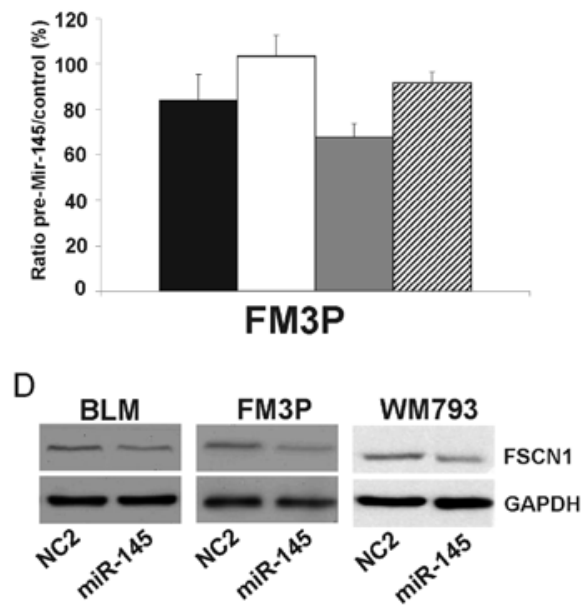

Figure 4. Effect of miR-145 upregulation on the expression of (potential) target genes. In order to examine the effect of miR-145 on the expression of potential target genes, melanoma cell lines (A) BLM, (B) WM793 and (C) FM3P were transfected with $50 \mathrm{nM}$ pre-miR-145 or negative controls. The mean ratio (\%) of the treated samples versus the mean ratio of negative controls was plotted for each potential target gene. A significant decrease in gene expression level (ratio $<100 \%$ ) was observed for the genes, MYO5A and $F S C N 1$, in the BLM and WM793 cells and for RAB27A in the BLM cells only. The ratio (treated versus control) is shown as the means \pm SEM of three independent experiments. P-values were determined with an unpaired t-test $\left({ }^{* *} \mathrm{P}<0.001\right.$ or $\left.{ }^{*} \mathrm{P}<0.05\right)$. (D) Western blots demonstrating the downregulation of FSCN1 (55 kDa) in pre-miR-145-transfected cells compared to negative controls, $60 \mathrm{~h}$ post-transfection. Glyceraldehyde-3-phosphate dehydrogenase (GAPDH, $36 \mathrm{kDa}$ ) was used as the loading control. The blots are representative of three independent experiments.

\section{Discussion}

Based on miRNA profiling studies from malignant melanoma tissues it is assumed that miR-145 plays a tumor suppressor role in melanoma $(8,22)$. Functional data unraveling the role of miR-145 in melanoma are limited. In this study, we used three human melanoma cell lines in which we examined the expression of miR-145. Our experiments suggest that miR-145 expression is biphasic, in that its expression is slightly increased 
A
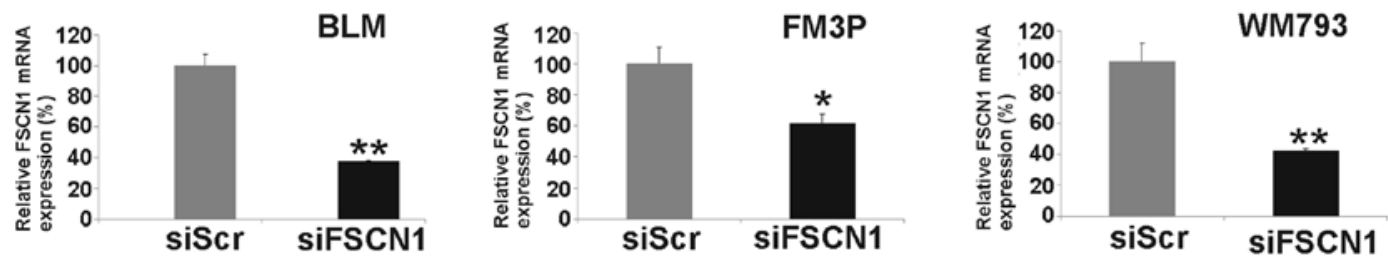

B
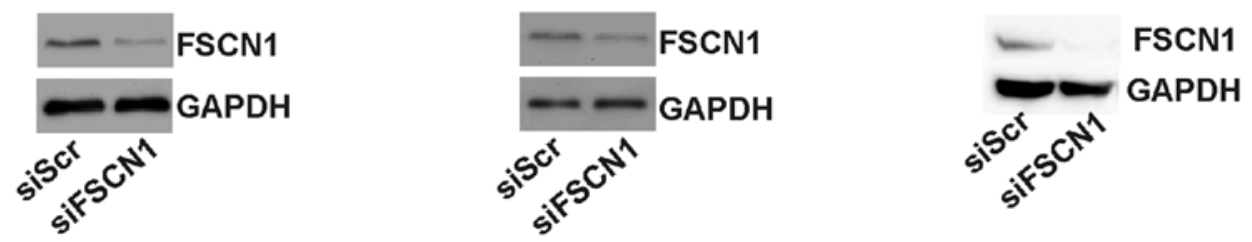

Figure 5. Knockdown of $F S C N 1$ in melanoma cell lines results in a decrease of $F S C N 1$, both at the mRNA and protein expression level. (A) Real-time qPCR was used to determine the relative expression level of FSCN1 following transfection of $50 \mathrm{nM}$ siFSCN1 or $50 \mathrm{nM}$ siScrambled (SiScr; negative control) into BLM, FM3P and WM793 cells. Significant decreases were observed in all three miR-145-transfected cell lines compared to their negative controls (100\%). The means of three independent experiments \pm SEM are shown. P-values were determined with an unpaired t-test $\left({ }^{* * *} \mathrm{P}<0.01\right.$ and $\left.{ }^{*} \mathrm{P}<0.05\right)$. (B) Western blots demonstrating the downregulation of FSCN1 (55 kDa) after the silencing of FSCN1, $60 \mathrm{~h}$ post-transfection. GAPDH was used as the loading control (36 kDa). The blots are representative of three independent experiments.

\section{A}

\section{Cell proliferation assay}
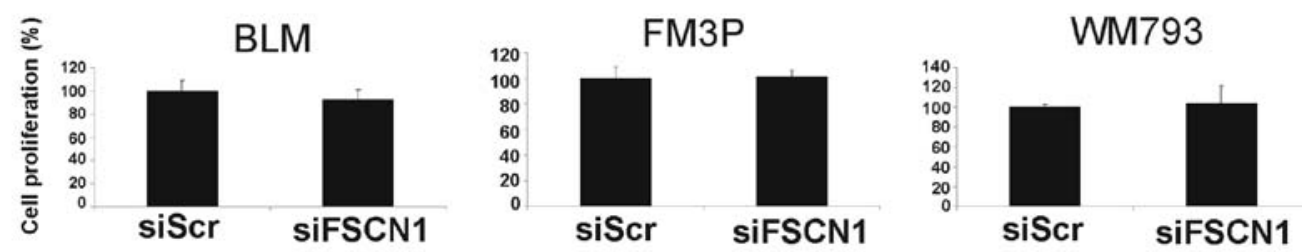

\section{B \\ Wound healing assay}

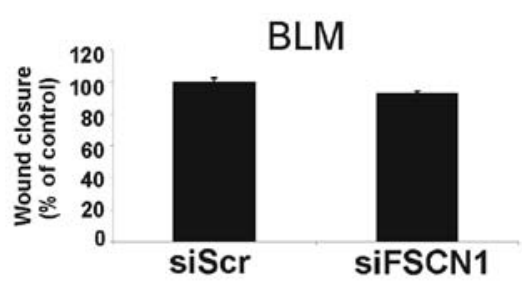

$\mathrm{Oh}$

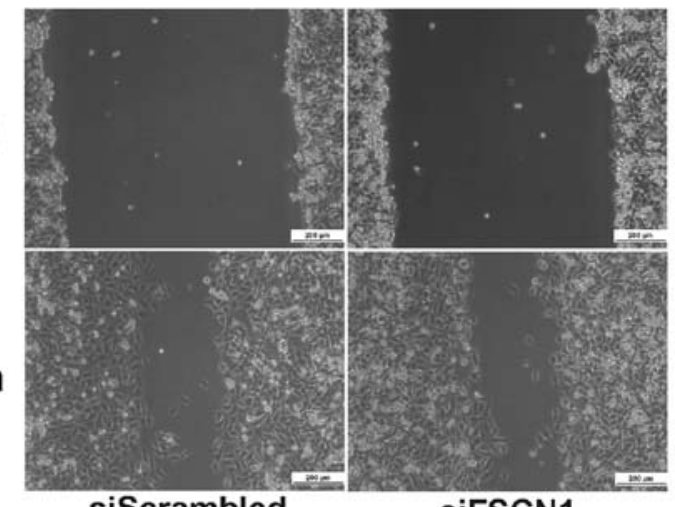

siScrambled

siFSCN1

Figure 6. Knockdown of FSCN1 does not alter cell proliferation and migration in the melanoma cell lines, BLM, FM3P and WM793. (A) The number of viable cells was counted $48 \mathrm{~h}$ following transfection of $50 \mathrm{nM}$ siFSCN1 or scrambled siRNA in each melanoma cell line. No effect on cell growth was observed. (B) Cell migration activity was determined by the wound healing assay. The results for the migration assay for BLM cells are presented as a histogram (left panel) and as phase contrast images (right panel) (scale bar, $200 \mu \mathrm{m}$ ). No difference in migration was observed for the siFSCN1-transfected BLM cells versus the siScrambled-transfected cells $(100 \%)$. The means of three independent experiments \pm SEM are depicted. siScr, siScrambled.

in primary, non-metastatic melanoma cells compared to normal melanocytes and is significantly reduced as the cancer progresses from non-metastatic to metastatic. Furthermore, our data suggest diverse functions of miR-145 among different melanoma cell lines, depending on the invasive capacity. This hypothesis was supported by our in vitro experiments using miR-145 mimics. In primary, non-invasive melanoma cells, miR-145 mimics exerted an anti-proliferative effect (tumor suppressor response), whereas in metastatic melanoma cells, migration and matrix invasion were suppressed by miR-145 
Invasion assay

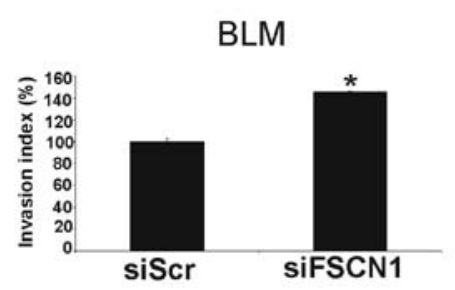

FM3P
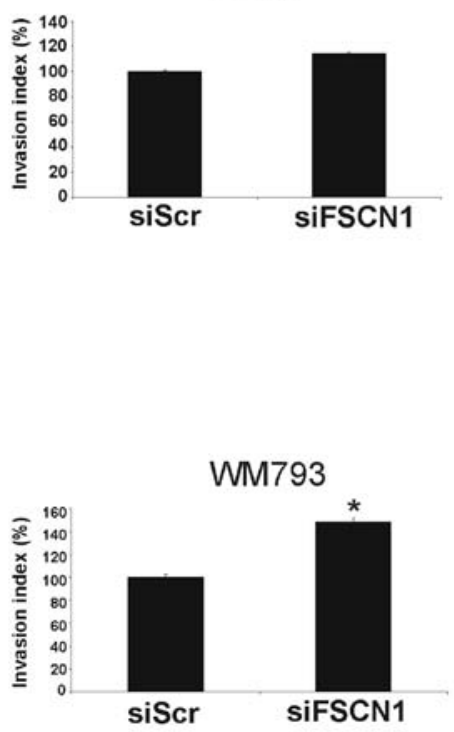
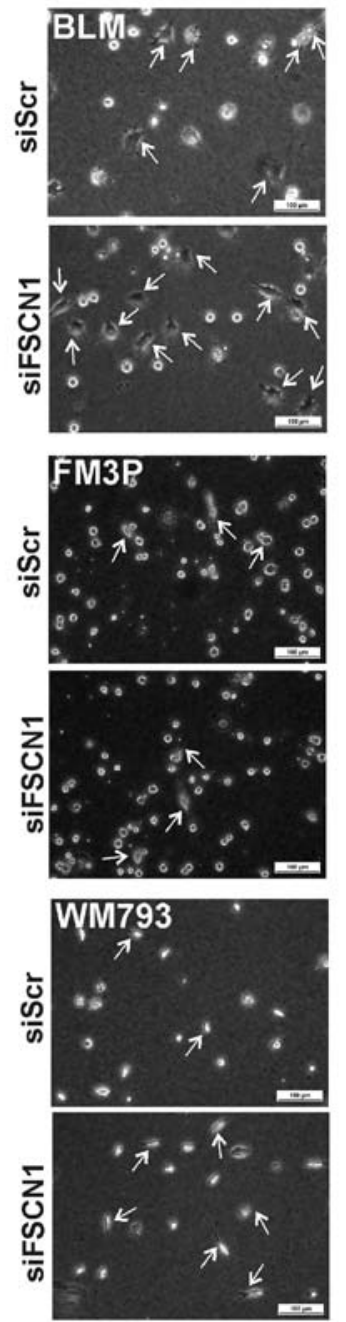

Figure 7. Knockdown of FSCN1 increases cell invasion in melanoma cell lines. Invasion assays were performed following the transfection of siFSCN1 and scrambled siRNA (siScrambled; siScr) into melanoma cell lines. The silencing of FSCN1 induced a significant increase in the invasion of BLM and WM793 cells. The invasion index (\%) is depicted on the Y-axis of each histogram (left panel) and is rescaled to $100 \%$ in the negative controls (siScr) Phase contrast images are shown on the right panel. Arrows indicate invasive extensions (scale bar, $100 \mu \mathrm{m}$ ). P-values were determined by the MannWhitney test $\left({ }^{*} \mathrm{P}<0.001\right)$.

overexpression (invasion suppressor response). A similar phenomenon was observed by Sachdeva et al while studying the effect of miR-145 on cell growth in non-metastatic MCF-7 breast cancer cells versus two other metastatic breast cancer cell lines $(23,24)$.

The observed miR-145 effects may be regulated through the modulation of the expression of genes essential for the formation of an invasive front. To determine the mechanims by which miR-145 exerts its invasion suppressor function, we investigated the expression of several target genes (FSCN1, $M Y O 5 A$ and $S O X 9$ ) and an indirect target (RAB27A) following the overexpression of miR-145 in the melanoma cell lines. The expression of $S O X 9$, recently reported as a miR-145 target in mouse mesenchymal stem cells (25), was not significantly reduced at the mRNA level following the ectopic expression of miR-145. Therefore, we did not focus any further on

this gene. The mRNA expression of MYO5A, however, was reduced following the overexpression of miR-145 in our melanoma cell lines. We recently demonstrated that MYO5A was a direct target of miR-145 by luciferase assays and functional studies in pigment cells (18). In addition, we reported that the downregulation of MYO5A following miR-145 overexpression also resulted in the reduced expression of RAB27A in normal primary melanocytes (18). Both proteins are part of a tripartite complex (MYO5A-RAB27A-MLPH) involved in the intracellular transport of melanosomes in pigment cells. Loss of one of the members of this tripartite complex results in its destabilization (14). To our knowledge, the presence of a functional MYO5A-RAB27A(-MLPH) complex in melanoma has not been shown to exist thus far. Due to the low or absent protein expression of MYO5A and RAB27A in our studied melanoma cell lines, compared to normal human melanocytes, we were unable to perform further functional assays to examine the effects of MYOVA (or RAB27A) knockdown in melanoma cells. Consequently, this led to the assumption that miR-145 does not exert its invasion suppressor function through the direct targeting of MYO5A, which is mainly involved in melanogenesis, or the disruption of the MYO5A-RAB27A(-MLPH) melanosomal pathway, which is probably not present or functional in this set of studied melanoma cell lines.

Of note, mRNA and protein expression levels of FSCN1 were reduced in all three studied melanoma cell lines following the overexpression of miR-145. FSCN1 is an actinbinding protein required for the formation of actin-based cell-surface protrusions that mediate interactions between cells and the extracellular matrix, cell-to-cell interactions and cell migration; it is required for the formation of cytoplasmic bundles of microfilaments that contribute to cellular architecture and movement $(26,27)$. Several studies have already reported that miR-145 targets FSCN1 in different human cancer types. In these cancers, the loss of miR-145 has been shown to promote the upregulation of FSCN1 expression, contributing to oncogenesis and tumor progression $(19,20)$. Based on this knowledge, as well as on our obtained expression data, we further focused on the role of FSCN1 in our melanoma cell lines. The knockdown of FSCN1 did not affect cell growth in these cell lines. These observations are in accordance with those reported by Noguchi et al (11) and point to the fact that FSCN1 does not affect cell proliferation. In contrast to the latter study, the silencing of FSCN1 did not result in reduced cell migration in our studied melanoma cell lines, further suggesting that miR-145 exerts its invasion suppressor role independently of $F S C N 1$, even when the expression level of FSCNI was reduced following the overexpression of miR-145. In this study, the effect of FSCN1 knockdown on cell invasion was studied for the first time in melanoma cell lines. Surprisingly, we found that the silencing of FSCN1 resulted in an increase in cell invasiveness, and thus metastatic potential, in two out of the three studied melanoma cell lines, namely BLM and WM793. As already mentioned, FSCN1 expression is often highly upregulated in tumors compared to normal matching tissues which exhibit no FSCN1 expression. The high expression of FSCN1 is associated with metastasis and poor prognosis in these types of cancer. In melanoma, however, FSCN1 seems to follow a different expression pattern. Yildiz et al (28) 
demonstrated by immunohistochemical analysis that FSCN1 was less frequently expressed in malignant melanoma (including metastatic melanoma) compared to benign and dysplastic nevi. These results are in accordance with those reported by Goncharuk et al (29), who performed immunohistochemical staining for FSCN1 in different skin neoplasias. They concluded that almost all melanocytic nevi expressed FSCN1, while no or weak FSCN1 expression was present in melanomas with pagetoid intra-epidermal spread and in invasive tumors with a high metastatic risk. The expression of FSCN1 decreases as formation and progression stage of melanoma advances. Both studies concluded that the loss of FSCN1 contributes to the development of invasive and metastatic phenotypes of melanoma. The downregulation or loss of the actin-bundling properties of FSCN1, which is probably associated with the disorganization of cell-cell and cell-matrix interactions, would stimulate cell motility and be an important step in the progression from locally invasive to widely disseminated melanoma. Our in vitro data point out that the loss of FSCN1 stimulates invasiveness and correspond with the conclusions obtained from these in vivo studies. It is clear from these data that FSCN1 plays a different role in melanoma than in other tumor types. Instead of exerting an oncogenic function it may be a metastasis suppressor gene involved in reducing invasive potential in melanoma. We hypothesize that its expression in melanoma is possibly regulated by miRNAs different from miR-145, as a low expression of FSCN1 in metastatic melanoma would correspond with a high expression of miR-145, which is not the case based on previous reports $(11,22)$ and on our in vitro data. Further research, aimed towards determining the expression levels of miR-145 and FSCN1 in different stages of melanoma, is warranted to support this hypothesis.

In conclusion, our results provide additional evidence that miR-145 acts as an invasion suppressor in malignant melanoma; when lost, tumor progression and metastatic potential are stimulated. Reintroduction of miR-145 could possibly reduce the invasiveness or metastatic potential of melanomas, making miR-145 potentially useful in the therapy of melanoma, either alone or in combination with other existing treatment regimens. Future research is warranted, using in vivo models with suitable (topical) delivery systems. The mechanism of action of miR-145 or its regulatory effect on target genes and pathways in melanoma remain unclear. SOX9 and MYO5A were not involved in the biological effects caused by miR-145 mimics. In addition, we illustrated that miR-145 does not necessarily act via the target FSCN1 in melanoma, in contrast to other tumor types, which indicates that other miR-145 target genes, such as MUC1, MMP-11, FLII and JAM-A $(24,30,31)$ or pathways independent of FSCN1 are involved in cell migration and invasion. Further research is required to clarify these observations.

\section{Acknowledgements}

We thank Martine De Mil for her assistance with cell culturing and Marie-Chantal Herteleer for her technical assistance. We thank Wendy De Rycke for her assistance with the collagen I invasion assays.

\section{References}

1. Garbe $\mathrm{C}$ and Leiter U: Melanoma epidemiology and trends. Clin Dermatol 27: 3-9, 2009.

2. Chin L: The genetics of malignant melanoma: lessons from mouse and man. Nat Rev Cancer 3: 559-570, 2003.

3. Lin K, Baritaki S, Militello L, Malaponte G, Bevelacqua Y and Bonavida B: The role of B-RAF mutations in melanoma and the induction of EMT via dysregulation of the NF- $\kappa$ B/Snail/RKIP/PTEN circuit. Genes Cancer 1: 409-420, 2010.

4. Howell PM Jr, Li X, Riker AI and Xi Y: MicroRNA in melanoma. Ochsner J 10: 83-92, 2010.

5. Mueller DW and Bosserhoff AK: Role of miRNAs in the progression of malignant melanoma. Br J Cancer 101: 551-556, 2009.

6. Mueller DW and Bosserhoff AK: The evolving concept of 'melano-miRs'-microRNAs in melanomagenesis. Pigment Cell Melanoma Res 23: 620-626, 2010.

7. Molnár V, Tamási V, Bakos B, Wiener Z and Falus A: Changes in miRNA expression in solid tumors: an miRNA profiling in melanomas. Semin Cancer Biol 18: 111-122, 2008.

8. Segura MF, Belitskaya-Lévy I, Rose AE, et al: Melanoma microRNA signature predicts post-recurrence survival. Clin Cancer Res 16: 1577-1586, 2010.

9. Sachdeva M and Mo YY: miR-145-mediated suppression of cell growth, invasion and metastasis. Am J Transl Res 2: 170-180, 2010.

10. Iorio MV, Ferracin M, Liu CG, et al: MicroRNA gene expression deregulation in human breast cancer. Cancer Res 65: 7065-7070, 2005.

11. Noguchi S, Mori T, Hoshino Y, et al: Comparative study of anti-oncogenic microRNA-145 in canine and human malignant melanoma. J Vet Med Sci 74: 1-8, 2012.

12. Kirkin AF, Petersen TR, Olsen AC, Li L, thor Straten P and Zeuthen J: Generation of human-melanoma-specific $\mathrm{T}$ lymphocyte clones defining novel cytolytic targets with panels of newly established melanoma cell lines. Cancer Immunol Immunother 41: 71-81, 1995.

13. Naeyaert JM, Eller M, Gordon PR, Park HY and Gilchrest BA: Pigment content of cultured human melanocytes does not correlate with tyrosinase message level. Br J Dermatol 125: 297-303, 1991.

14. Van Gele M, Geusens B, Schmitt AM, Aguilar L and Lambert J: Knockdown of myosin Va isoforms by RNAi as a tool to block melanosome transport in primary human melanocytes. J Invest Dermatol 128: 2474-2484, 2008.

15. Vandesompele J, De Paepe A and Speleman F: Elimination of primer-dimer artifacts and genomic coamplification using a two-step SYBR green I real-time RT-PCR. Anal Biochem 303: 95-98, 2002.

16. Vandesompele J, De Preter K, Pattyn F, et al: Accurate normalization of real-time quantitative RT-PCR data by geometric averaging of multiple internal control genes. Genome Biol 3: Research0034, 2002.

17. De Wever O, Hendrix A, De Boeck A, et al: Modeling and quantification of cancer cell invasion through collagen type I matrices. Int J Dev Biol 54: 887-896, 2010.

18. Dynoodt P, Mestdagh P, Van Peer G, et al: Identification of miR-145 as a key regulator of the pigmentary process. J Invest Dermatol: August 16, 2012 (E-pub ahead of print). doi:10.1038/ jid.2012.266

19. Chiyomaru T, Enokida H, Tatarano S, et al: miR-145 and miR-133a function as tumour suppressors and directly regulate FSCN1 expression in bladder cancer. Br J Cancer 102: 883-891, 2010.

20. Fuse M, Nohata N, Kojima S, et al: Restoration of miR-145 expression suppresses cell proliferation, migration and invasion in prostate cancer by targeting FSCN1. Int J Oncol 38: 1093-1101, 2011.

21. Kim SJ, Oh JS, Shin JY, et al: Development of microRNA-145 for therapeutic application in breast cancer. J Control Release 155: 427-434, 2011.

22. Mascellani N, Tagliavini L, Gamberoni G, et al: Using miRNA expression data for the study of human cancer. Minerva Biotec 20: 23-30, 2008

23. Sachdeva M, Zhu S, Wu F, et al: p53 represses c-Myc through induction of the tumor suppressor miR-145. Proc Natl Acad Sci USA 106: 3207-3212, 2009. 
24. Sachdeva M and Mo YY: MicroRNA-145 suppresses cell invasion and metastasis by directly targeting mucin 1 . Cancer Res 70: 378-387, 2010.

25. Yang B, Guo H, Zhang Y, Chen L, Ying D and Dong S: MicroRNA-145 regulates chondrogenic differentiation of mesenchymal stem cells by targeting Sox9. PLoS One 6: e21679, 2011.

26. Kureishy N, Sapountzi V, Prag S, Anilkumar N and Adams JC: Fascins, and their roles in cell structure and function. Bioessays 24: 350-361, 2002.

27. Hashimoto Y, Skacel M and Adams JC: Roles of fascin in human carcinoma motility and signaling: prospects for a novel biomarker? Int J Biochem Cell Biol 37: 1787-1804, 2005.
28. Yildiz L, Kefeli M, Aydin O and Kandemir B: Fascin expression in melanocytic lesions of the skin. Eur J Dermatol 19: 445-450, 2009.

29. Goncharuk VN, Ross JS and Carlson JA: Actin-binding protein fascin expression in skin neoplasia. J Cutan Pathol 29: 430-438, 2002.

30. Zhang J, Guo H, Zhang H, et al: Putative tumor suppressor miR-145 inhibits colon cancer cell growth by targeting oncogene Friend leukemia virus integration 1 gene. Cancer 117: 86-95, 2011.

31. Götte M, Mohr C, Koo CY, et al: miR-145-dependent targeting of junctional adhesion molecule $\mathrm{A}$ and modulation of fascin expression are associated with reduced breast cancer cell motility and invasiveness. Oncogene 29: 6569-6580, 2010. 